\title{
Classification and properties of UV extinction curves
}

\author{
G. Barbaro ${ }^{1}$, P. Mazzei $^{2}$, L. Morbidelli ${ }^{3}$, P. Patriarchi ${ }^{3}$, and M. Perinotto ${ }^{4}$ \\ 1 Dipartimento di Astronomia, Vicolo Osservatorio 3, 35122 Padova, Italy \\ 2 Osservatorio Astronomico, Vicolo Osservatorio 5, 35122 Padova, Italy \\ 3 CAISMI-CNR, Firenze, Italy \\ 4 Dipartimento di Astronomia, Firenze, Italy
}

Received 15 February 2000 / Accepted 2 October 2000

\begin{abstract}
The catalog of Savage et al. (1985) reporting colour excesses of 1415 stars from ANS photometry offers the opportunity to deeply investigate the characteristics of UV extinction curves which differ from the standard extinction of the diffuse interstellar medium. To this aim we have selected a sample of 252 curves, which have been compared with the relations derived by Cardelli et al. (1989; CCM in the following) for a variety of $R_{V}$ values in the range 2.4-5 and have been classified as normal if they fit at least one of the CCM curves or anomalous otherwise. We find that normal curves with small $R_{V}$ are just as numerous as those with large $R_{V}$. The anomalous objects are arranged into two groups according to the strength of the bump at $0.217 \mu$. For a given value of $c_{2}$ this increases along the sequence: type A anomalous, normals and type B anomalous, suggesting that this sequence should correspond to an increase of the amount of small grains along the sightline. Considerations concerning the environmental characteristics indicate that the anomalous behaviour is not necessarily tied to the existence of dense gas clouds along the line of sight.
\end{abstract}

Key words. stars: circumstellar matter - ISM: dust, extinction - ISM: general

\section{Introduction}

It has been recognized that a single interstellar extinction curve (standard curve; Seaton 1979; Savage \& Mathis 1979) is able to represent the behaviour of the dust extinction towards several directions across the galactic disk. The meaning of such a curve in terms of the responsible grains and their physical properties has been extensively studied in various fundamental papers (Mathis et al. 1977; Draine \& Lee 1984; Désert et al. 1990; Mathis 1994; Li \& Greenberg 1997; Mathis 1998) suggesting basic explanations in terms of two or three different types of particles. It was also shown that the standard curve characterizes the behaviour of the dust along sightlines crossing a diffuse environment devoid of dense gas. The extinction in an interstellar medium with different physical properties deviates from the standard extinction. A well known example is offered by the case of $\theta^{1}$ Ori $\mathrm{C}$ characterized by a considerably smaller extinction in the UV. Several other extinction curves have been singled out and studied which display a variety of shapes especially in the UV region.

Cardelli et al. (1988) have analysed a sample of 31 extinction curves and found that they constitute a monoparametric family with respect to $R_{V}$. However lines

Send offprint requests to: G. Barbaro of sight are also found which deviate, beyond the uncertainty of the observations, from the relations of Cardelli et al. (1989), e.g. Cardelli \& Clayton (1991), Mathis \& Cardelli (1992). This discrepancy is considerable in some cases extensively analysed: HD 29647 (Snow \& Seab 1980; Cardelli \& Savage 1988), HD 62542 (Cardelli \& Savage 1988; Cardelli et al. 1990), HD 204827 (Cardelli \& Clayton 1991), HD 210121 (Welty \& Fowler 1999; Larson et al. 1996), VCT 10 (Cardelli \& Clayton 1991).

The catalog of Savage et al. (1985) allows to study, extensively, the properties of the UV extinction curves. In the following we present the results of an analysis performed on a subsample of curves of this catalog. On the basis of the comparison with the CCM relations, they have been subdivided into two classes: the "normal" and "anomalous" ones, the former being those which fit and the latter those which do not fit these relations.

\section{Selection of the sample and classification of the curves}

From the ANS UV photometric catalog of Wesselius et al. (1982), Savage et al. (1985) derived a list of colour excesses $E(33-V), E(25-V), E(22-V), E(18-V)$, $E(15-V)$ in the direction of 1415 stars. From these data 
Table 1. Number of objects in the different intervals of $E(B-V)$ and $D^{2}$

\begin{tabular}{|c|c|c|c|c|c|}
\hline & \multicolumn{5}{|c|}{$D^{2} \geq 0 . D^{2} \geq 1 . D^{2} \geq 2 . D^{2} \geq 3 . D^{2} \geq 4$} \\
\hline$E(B-V) \geq 0.1$ & 941 & 532 & 304 & 191 & 133 \\
\hline$E(B-V) \geq 0.2$ & 795 & 448 & 252 & 156 & 105 \\
\hline$E(B-V) \geq 0.3$ & 678 & 364 & 195 & 117 & 78 \\
\hline$E(B-V) \geq 0.4$ & 542 & 278 & 136 & 78 & 52 \\
\hline$E(B-V) \geq 0.5$ & 418 & 211 & 95 & 52 & 35 \\
\hline$E(B-V) \geq 0.6$ & 296 & 147 & 65 & 36 & 25 \\
\hline$E(B-V) \geq 0.7$ & 193 & 102 & 51 & 31 & 25 \\
\hline$E(B-V) \geq 0.8$ & 120 & 68 & 34 & 21 & 17 \\
\hline$E(B-V) \geq 0.9$ & 67 & 38 & 25 & 17 & 13 \\
\hline$E(B-V) \geq 1.0$ & 32 & 19 & 14 & 10 & 6 \\
\hline
\end{tabular}

the normalized extinction curves $\varepsilon(\lambda)$ are easily derived by dividing by $E(B-V)$.

The ANS photometry is generally very accurate and for stars with errors smaller than 0.2 mag the extinction curves are in good agreement with those derived by the IUE satellite (Massa et al. 1983). The most relevant errors are those due to the mismatch of the spectral classes of the star under examination and the comparison unreddened star. By adopting an uncertainty of one spectral subclass Meyer \& Savage (1981) estimate for spectral types in the range B0-B7 and luminosity class $V$ errors $\Delta(\lambda-V)$ in $\varepsilon(\lambda)$ smaller than few tenths. The errors are larger for stars later than B7 and for supergiants.

Savage et al. (1985) give also the differences $D_{15}$ and $D_{\mathrm{b}}$ between each curve and the average curve of the sample at $\lambda=0.15 \mu$ and at $\lambda=0.22 \mu$ (the wavelength corresponding to the maximum of the bump) respectively, normalized to their mean square deviation. The quantity $D^{2}=D_{\mathrm{b}}^{2}+D_{15}^{2}$ marks out the discrepancy of every curve from the average curve of the sample, which is very similar to the standard extinction curve with $R_{V}=3.1$. Savage et al. (1985) claim that $43 \%$ of the sample stars with $E(B-V)>0.20$ exhibits strong deviations from the average curve.

We wish to further investigate this aspect. To this aim we have selected, from the catalog of Savage et al. (1985), the objects with extinction curves very different from the standard one, namely those with a large $D^{2}$. In doing this we have excluded objects lacking $D_{\mathrm{b}}^{2}$ and $D_{15}^{2}$ and/or with negative $E(\lambda-V)$. Since in the derivation of $\varepsilon(\lambda)$ the division by $E(B-V)$ gives rise to large errors when $E(B-V)$ is small, it is worthwhile to exclude the cases with small reddening. We have then retained only stars with $E(B-V) \geq 0.20$.

Table 1 shows the number of objects in different ranges of $E(B-V)$ and $D^{2}$.

Moreover in selecting our sample we have adopted the constraint $D^{2} \geq 2$.

In this way we have obtained a subsample of 252 objects corresponding to about $18 \%$ of the original sample.
A classification has been defined based on the congruence with the relations derived by CCM:

$$
\begin{aligned}
& \varepsilon(x)=R_{V}\left[\frac{A(\lambda)}{A(V)}-1\right] \\
& \frac{A(\lambda)}{A(V)}=a(x)+b(x) \frac{1}{R_{V}}
\end{aligned}
$$

with $x=1 / \lambda \mu^{-1}$. Each curve of our sample $\varepsilon_{i}\left(x_{\mathrm{r}}\right)$ has been compared with the extinction curves $\varepsilon_{j}\left(x_{\mathrm{r}}\right)$ computed with the previous relations for a series of $R_{V}$ values in the range $2.40-5.00$ and the sum of the squares of the differences has been computed:

$\Delta_{i, j}=\Sigma_{r}\left[\varepsilon_{i}\left(x_{\mathrm{r}}\right)-\varepsilon_{j}\left(x_{\mathrm{r}}\right)\right]^{2}$

where the summation is extended to the wavelengths considered in the catalog of Savage et al. (1985), namely to $x=2.27,3.04,4.00,4.55,5.56,6.67$. Each value of $j$ corresponds to a value of $R_{V}$.

From the analysis of the quantities $\Delta_{i, j}$ the following situations can arise for every curve:

- for every $R_{V}$ value, $\Delta_{i, j}$ is always larger than 1 . In this case we consider that the observed curve cannot be fitted by any CCM relation: we call such a curve anomalous.

- there exists at least one value of $R_{V}$ for which $\Delta_{i, j} \leq$ 0.5. We assume that there is a reasonable fit with a CCM curve and call such sightline normal. The value of $R_{V}$ which minimizes $\Delta_{i, j}$ gives an estimate of this parameter. It must be noted however that the smallest values of $R_{V}$ are obtained through an extrapolation since, in the sample used by CCM to derive their relation, $R_{V}$ does not go below 2.60 ;

- the minimum of $\Delta_{i, j}$ lies in the range $0.5-1$. Such sightlines will not be further considered.

Among the normal curves we distinguish: a) normal curves with $R_{V}$ large $(\geq 3.85)$; b) normal curves with $R_{V}$ small $(\leq 2.80)$; c) normal curves with $R_{V}$ intermediate $\left(2.80<R_{V}<3.85\right)$. Also this last group will be ignored in the following.

The stars with normal extinction curves, respectively with $R_{V}$ large and $R_{V}$ small, are listed in Tables 2 and 3, together with the corresponding $R_{V}$ values. There are 33 objects in both groups.

We have found a considerable number of extinction curves with small values of $R_{V}$ : curves of this type have been so far barely considered in the literature. Very small values of $R_{V}$ have been mentioned by Whittet \& van Breda (1980) who found that the smallest value of such parameter in a sample of about $200 \mathrm{OB}$ stars is 2.40 . Recently Szomoru \& Guhathakurta (1999) have analysed the behaviour of the extinction in four cirrus clouds by means of star counts and have inferred $R_{V}$ values much smaller than the standard value. Misselt et al. (1999) have studied the extinction in direction of several stars of the LMC and have determined very often values smaller than 
Table 2. Normal lines of sight with $R_{V}$ large

\begin{tabular}{lccccc}
\hline name & $\Delta_{i, j} \min$ & $R_{V}$ & name & $\Delta_{i, j} \min$ & $R_{V}$ \\
\hline HD 25833 & 0.43 & 4.25 & HD 147165 & 0.21 & 4.15 \\
HD 36629 & 0.44 & 4.15 & HD 147701 & 0.44 & 4.00 \\
HD 37356 & 0.16 & 4.50 & HD 147889 & 0.42 & 4.40 \\
HD 37903 & 0.43 & 4.60 & HD 147933 & 0.14 & 4.80 \\
HD 252325 & 0.20 & 4.65 & HD 149452 & 0.29 & 4.05 \\
HD 253327 & 0.21 & 3.85 & HD 159176 & 0.16 & 3.90 \\
HD 64315 & 0.44 & 3.85 & HD 166539 & 0.15 & 4.05 \\
HD 66546 & 0.27 & 4.10 & HD 166546 & 0.09 & 4.20 \\
HD 68633 & 0.40 & 4.35 & HD 167264 & 0.36 & 4.70 \\
HD 68982 & 0.14 & 4.35 & HD 168021 & 0.40 & 5.00 \\
HD 73420 & 0.37 & 4.50 & HD 168137 & 0.26 & 4.40 \\
HD 73849 & 0.40 & 4.20 & HD 168785 & 0.31 & 4.65 \\
HD 97966 & 0.35 & 3.95 & HD 344894 & 0.36 & 3.85 \\
HD 101008 & 0.30 & 4.20 & HD 345214 & 0.39 & 4.60 \\
HD 101298 & 0.31 & 3.85 & HD 211880 & 0.35 & 4.15 \\
HD 142096 & 0.12 & 4.25 & BD +62 2142 & 0.50 & 3.90 \\
HD 145792 & 0.10 & 4.90 & & & \\
\hline
\end{tabular}

Table 3. Normal lines of sight with $R_{V}$ small

\begin{tabular}{lccccc}
\hline name & $\Delta_{i, j} \min$ & $R_{V}$ & name & $\Delta_{i, j} \min$ & $R_{V}$ \\
\hline HD 3191 & 0.25 & 2.55 & HD 152218 & 0.41 & 2.75 \\
HD 5552 & 0.26 & 2.65 & HD 173694 & 0.39 & 2.65 \\
BD +60 232 & 0.02 & 2.40 & HD 175514 & 0.40 & 2.80 \\
HD 236923 & 0.29 & 2.45 & HD 176304 & 0.24 & 2.40 \\
BD +54 490 & 0.09 & 2.40 & HD 183561 & 0.37 & 2.50 \\
HD 14014 & 0.38 & 2.40 & HD 187851 & 0.26 & 2.45 \\
HD 14092 & 0.04 & 2.55 & HD 189779 & 0.33 & 2.75 \\
HD 14250 & 0.20 & 2.65 & HD 190429 & 0.11 & 2.40 \\
BD +56 549 & 0.11 & 2.40 & HD 192001 & 0.15 & 2.50 \\
HD 14501 & 0.25 & 2.70 & HD 192539 & 0.40 & 2.60 \\
HD 14956 & 0.31 & 2.55 & HD 197460 & 0.33 & 2.40 \\
HD 24432 & 0.44 & 2.40 & BD +45 3339 & 0.41 & 2.70 \\
BD +34 1150 & 0.26 & 2.65 & HD 206165 & 0.36 & 2.40 \\
BD +34 1162 & 0.22 & 2.80 & HD 216438 & 0.45 & 2.45 \\
HD 41690 & 0.09 & 2.45 & BD +61 2515 & 0.17 & 2.45 \\
HD 44139 & 0.23 & 2.55 & BD +66 1661 & 0.39 & 2.80 \\
HD 146332 & 0.26 & 2.55 & & & \\
\hline
\end{tabular}

3.1, which they ascribe to grains with dimensions smaller than the dust in our Galaxy.

The anomalous curves amount to 78. As already explained, their character is defined by the quantities $\Delta_{i, j}$; usually deviations from the CCM relation mainly occur in correspondence to $\lambda=0.22$ and $\lambda=0.18 \mu$. In some cases however the largest deviation is found at other wavelengths, in particular at $\lambda=0.33$ while in the region 0.17 $0.22 \mu$ they behave normally. These last curves have been also excluded. We believe that the colour excess of these curves at $\lambda=0.33 \mu$ is affected by significant uncertainties, as is evident e.g. in the case of HD $37022\left(\theta^{1}\right.$ Ori C) where the extinction value derived with ANS poorly compares with values derived with further observations, as the IUE satellite.

The curves of this type which have been excluded are those falling within the horizontal strip of Fig. 2, which will be mentioned further on.
Table 4. Anomalous lines of sight with the group to which they belong

\begin{tabular}{|c|c|c|c|c|c|}
\hline name & $\Delta_{i, j} \min$ & group & name & $\Delta_{i, j} \min$ & group \\
\hline HD 1337 & 3.81 & $\mathrm{~A}$ & HD 236589 & 1.01 & $\mathrm{~A}$ \\
\hline $\mathrm{BD}+57252^{*}$ & 3.81 & - & HD 232522 & 1.02 & A \\
\hline $\mathrm{BD}+62338$ & 1.16 & B & HD 13659 & 1.89 & A \\
\hline HD 14734 & 22.92 & A & HD 21455 & 4.19 & A \\
\hline HD 24912 & 1.58 & B & HD $25639^{*}$ & 11.15 & - \\
\hline HD 27192 & 1.01 & B & HD 32672 & 1.71 & B \\
\hline HD 33203 & 1.42 & A & HD 35215 & 1.78 & B \\
\hline HD 36841 & 13.11 & A & HD 37022 & 8.02 & A \\
\hline HD $37061^{*}$ & 12.39 & - & HD 37367 & 1.48 & B \\
\hline HD 37366 & 1.04 & B & HD $37767^{*}$ & 1.80 & - \\
\hline HD 39680 & 2.52 & A & HD 249845 & 1.29 & A \\
\hline HD 41831 & 1.19 & A & HD 254577 & 1.14 & A \\
\hline HD 46867 & 2.06 & A & HD 52559 & 16.49 & A \\
\hline HD 53755 & 1.54 & A & HD $60325^{*}$ & 1.75 & - \\
\hline HD 62542 & 5.24 & A & HD $71609^{*}$ & 1.03 & - \\
\hline HD 72648 & 2.43 & B & HD 73495 & 102.55 & A \\
\hline HD $93205^{*}$ & 2.59 & - & HD 94144 & 1.05 & A \\
\hline HD 96917 & 1.68 & A & HD $101205^{*}$ & 65.82 & - \\
\hline HD 116084 & 1.04 & A & HD 116852 & 1.18 & $\mathrm{~A}$ \\
\hline HD 137569 & 7.36 & A & HD 142758 & 2.12 & A \\
\hline HD 145502 & 2.62 & B & HD 147617 & 1.25 & A \\
\hline HD 148422 & 1.19 & A & HD 148878 & 2.89 & A \\
\hline HD 149038 & 1.53 & B & HD 152096 & 2.45 & B \\
\hline HD $152560^{*}$ & 40.43 & - & HD 152741 & 2.09 & B \\
\hline HD 158243 & 7.89 & A & HD 160993 & 3.30 & A \\
\hline HD 161653 & 3.41 & B & HD 164353 & 3.41 & A \\
\hline HD 164073 & 1.30 & A & HD 164402 & 1.22 & B \\
\hline HD $164492^{*}$ & 3.77 & - & $\mathrm{BD}-134927$ & 2.57 & A \\
\hline HD 169454 & 1.02 & A & HD 175876 & 1.32 & A \\
\hline HD 176819 & 1.07 & B & HD 185418 & 1.21 & B \\
\hline HD 225757 & 31.66 & A & HD 187879 & 6.33 & A \\
\hline HD 227242 & 2.27 & A & HD 190991 & 1.38 & A \\
\hline HD 191396 & 2.50 & A & HD $228101^{*}$ & 1.16 & - \\
\hline $\mathrm{BD}+373945$ & 2.59 & A & $\mathrm{BD}+354258$ & 1.32 & A \\
\hline HD 199579 & 1.04 & A & $\mathrm{BD}+453360$ & 1.52 & A \\
\hline HD 204827 & 1.89 & A & $\mathrm{BD}+473588$ & 1.45 & A \\
\hline HD 210072 & 6.11 & A & $\mathrm{BD}+523122$ & 1.05 & A \\
\hline $\mathrm{BD}+552770^{*}$ & 1.41 & - & HD 217919 & 1.66 & A \\
\hline HD 224257 & 1.47 & A & HD 224424 & 1.46 & A \\
\hline
\end{tabular}

* the asterisk marks the curves which we have excluded

The anomalous curves are listed in Table 4; the twelve ones which have been excluded are also marked.

To summarize, our analysis is based on the following categories of sightlines:

1) normal curves with $R_{V}$ large ( $\geq 3.85$ ) (33 objects);

2 ) normal curves with $R_{V}$ small ( $\leq 2.85$ ) (33 objects);

3) anomalous curves (66 objects).

We are thus left with 132 sightlines out of the original 252 .

\section{The anomalous sightlines}

The diagram of Fig. 5 of Cardelli (1994) allows a clear separation of normal and anomalous curves. The same purpose can be reached with the diagram of Fig. 1 
Table 5. Sightlines plotted in Fig. 1

\begin{tabular}{lccccc}
\hline name & $(\Delta \epsilon)_{\text {oss }}$ & $R_{V}$ & $(\Delta \epsilon)_{\mathrm{CCM}}$ & $E(B-V)$ & $\sigma(\Delta \epsilon)_{\text {oss }}$ \\
\hline HD 37674 & 3.75 & 2.95 & 1.91 & 0.14 & 1.23 \\
HD 102065 & 3.75 & 3.90 & 2.54 & 0.17 & 1.02 \\
HD 103536 & 3.73 & 3.80 & 2.02 & 0.17 & 1.02 \\
HD 103875 & 3.65 & 3.20 & 1.95 & 0.32 & 0.54 \\
HD 38087 & 3.37 & 5.30 & 2.19 & 0.33 & 0.52 \\
HD 73882 & 0.87 & 3.39 & 1.97 & 0.71 & 0.24 \\
HD 204827 & 0.68 & 2.40 & 1.86 & 1.11 & 0.16 \\
HD 94414 & 0.43 & 3.90 & 2.54 & 0.67 & 0.26 \\
HD 98143 & 0.27 & 3.20 & 1.94 & 0.65 & 0.26 \\
HD 210121 & 0.17 & 2.10 & 1.81 & 0.40 & 0.43 \\
VCT 10 & -0.34 & 4.30 & 2.08 & 0.74 & 0.23 \\
HD 29647 & -0.50 & 3.60 & 2.00 & 1.01 & 0.17 \\
HD 62542 & -0.64 & 3.24 & 1.96 & 0.33 & 0.52 \\
\hline
\end{tabular}

in which the difference $(\Delta \epsilon)_{\text {obs }}$ between the observed extinctions $\epsilon(22)$ and $\epsilon(18)$ is plotted against the same quantity $(\Delta \epsilon)_{\mathrm{CCM}}$ as it results from CCM relations. Of course, since the evaluation of the latter quantity requires the knowledge of $R_{V}$, the plot of Fig. 1 concerns only sightlines with known $R_{V}$. The used stars are presented in Table 5 . Errors are evaluated following Massa \& Savage (1984).

The star symbol at the center of the diagram marks the average position of the normal sightlines of our sample (66 objects) with its standard deviation. In the upper and in the lower part of the diagram the anomalous curves are located. They define two distinct groups, separate from the mean of normal objects by at least $2 \sigma$. From this figure we can conclude that the discriminant parameter is only $(\Delta \epsilon)_{\text {obs }}$ and therefore we can extend the analysis to all the objects of our subsample using a $(\Delta \epsilon)_{\text {obs }}-\epsilon(18)$ diagram, which does not imply the knowledge of $R_{V}$. This diagram is shown in Fig. 2. Again the anomalous curves are separated from the normal ones and are arranged into two groups. When one takes into account errors due to the observations one cannot strictly maintain for all the objects of these groups the separation from normals. We believe however that, at least on average, the two groups of anomalous are distinct from the normals and surely all the elements of each group are separated from those of the other. The difference between the two groups originates from the strength of the bump: a group (A) with a bump weaker than the normal curves, including almost all the anomalous curves studied in literature, and another group (B) with a stronger bump. This is evident when we plot on both diagrams the loci $c_{2}=$ const and $c_{3} / \gamma^{2}=$ const.

In order to determine their equations we start with the the parameterization of Fitzpatrick \& Massa (1986, 1988; FM in the following):

$\varepsilon(x)=c_{1}+c_{2} x+c_{3} D(x)+c_{4} F(x)$

with

$D(x)=\frac{x^{2}}{\left(x^{2}-x_{\mathrm{o}}^{2}\right)^{2}+\gamma^{2} x^{2}}$

and with $F(x)=0$ for $x<5.9 \mu^{-1}$.

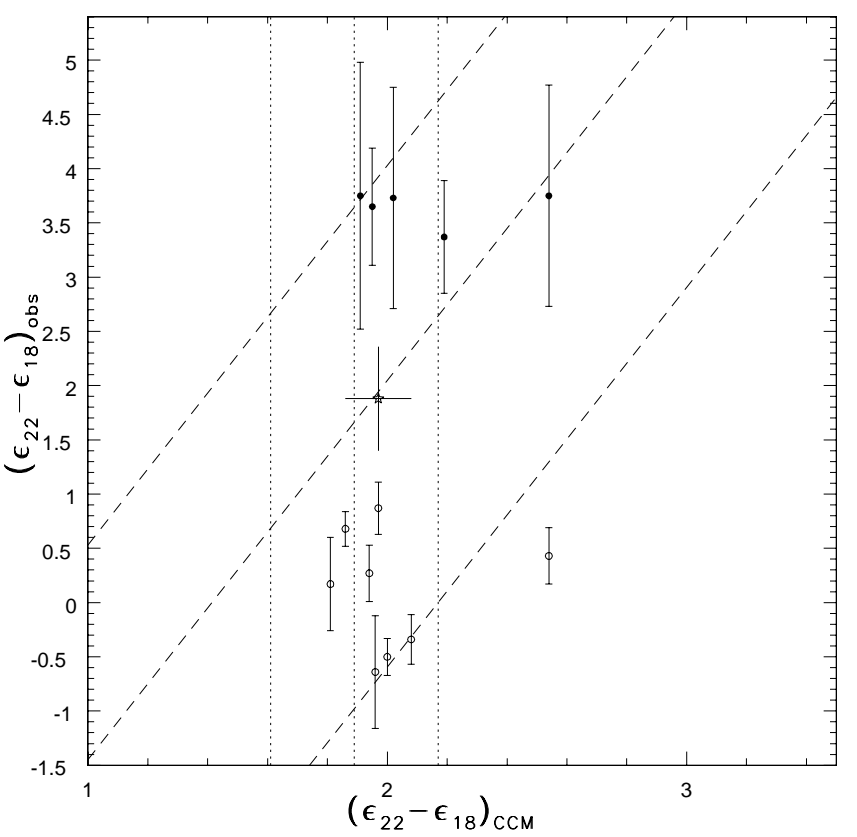

Fig. 1. Type A anomalous are shown with open circles, type $\mathrm{B}$ with filled circles; the star marks the average position of normal sightlines; loci $c_{2}=$ const $(2,1$ and 0 from the left to the right; dotted lines) and $c_{3} / \gamma^{2}=$ const $(7,4$, and 0 from the left to the right; dashed lines) are also plotted

Six parameters enter these relations: $c_{1}, c_{2}, c_{3}, c_{4}$, $x_{\mathrm{o}}$ and $\gamma$; one of them $\left(c_{4}\right)$ does not enter in the forthcoming discussion since our considerations concern wavelengths larger than $0.17 \mu\left(x<5.9 \mu^{-1}\right)$. Moreover another parameter, $c_{1}$, is not relevant, as Jenniskens \& Greenberg (1993) out of a sample of 115 extinction curves have found that $c_{1}$ and $c_{2}$ are linearly correlated. A similar result was also obtained by Carnochan (1986), Fitzpatrick \& Massa $(1986,1988)$ and CCM.

Relation (5) can be written:

$D(x)=\gamma^{-2}[1+d(x)]^{-1}$

with

$d(x)=\left(\frac{x^{2}-x_{\mathrm{o}}^{2}}{\gamma x}\right)^{2}$

In computing $\varepsilon_{22}$ one can adopt the following approximation:

$\left[1+d\left(x_{22}\right)\right]^{-1} \approx 1$

An inspection to the values of the FM parameters in a large sample of sightlines (see for instance Jenniskens \& Greenberg 1993) shows that prevalently the spread around this value is restricted to within few percents. Similarly with reference to $\varepsilon_{18},\left[1+d\left(x_{18}\right)\right]^{-1}$ essentially falls within the interval $0.20-0.50$. We have adopted the value $1 / 3$ :

$\left[1+d\left(x_{18}\right)\right]^{-1}=\frac{1}{3}$. 


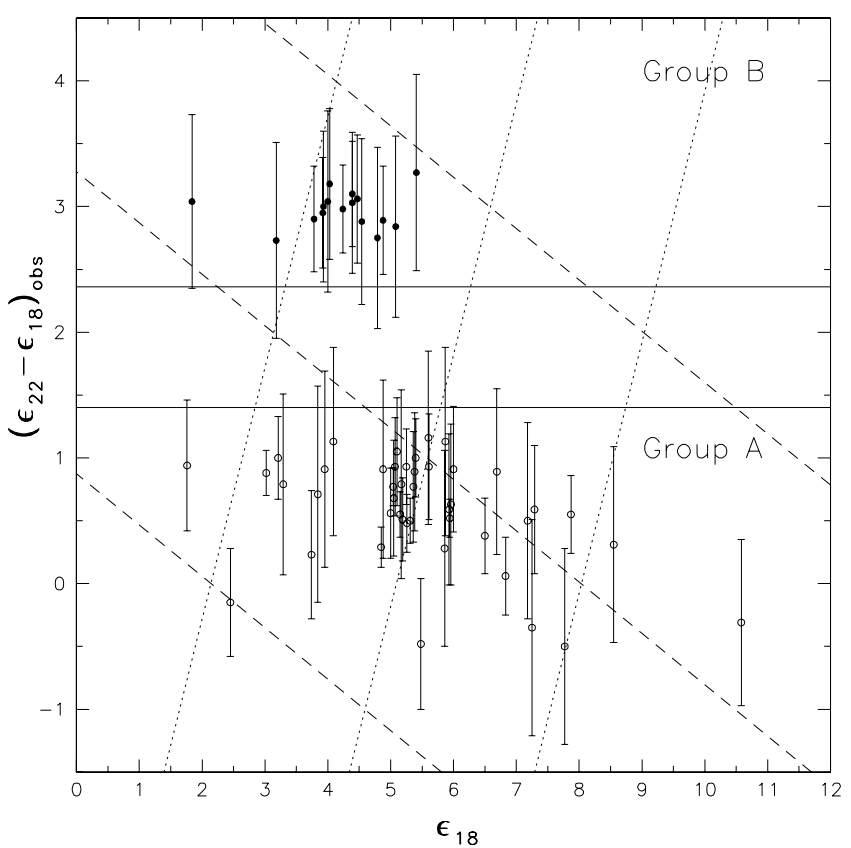

Fig. 2. Symbols are the same as in Fig. 1; the horizontal strip bounds the position of normal curves; the values of $c_{2}$ are 0,1 and 2 from the left to the right, those of $c_{3} / \gamma^{2}$ are 0,3 , and 5 respectively

Therefore one has:

$\varepsilon_{22}=\varepsilon\left(x_{22}\right) \approx c_{1}+c_{2} x_{22}+\frac{c_{3}}{\gamma^{2}}$

$\varepsilon_{18}=\varepsilon\left(x_{18}\right) \approx c_{1}+c_{2} x_{18}+\frac{1}{3} \frac{c_{3}}{\gamma^{2}}$.

Consequently, at least approximately and with regard to the considered wavelength range, the extinction curves form a biparametric system with parameters the slope $c_{2}$ of the linear background and the strength $c_{3} / \gamma^{2}$ of the bump. The relation $R_{V}=5.1-2.4 c_{2}$ of Cardelli et al. (1988) has been adopted in order to express $(\Delta \epsilon)_{\mathrm{CCM}}$. In this way the curves $c_{2}=$ const and $c_{3} / \gamma^{2}=$ const can be drawn in both Figs. 1 and 2, confirming that the two groups of anomalous sightlines are characterized by a different intensity of the bump compared with the linear background.

The existence of the anomalous curves, both of group A and group B, suggests that different environments cause a different behaviour of the grains.

Table 4, previously presented with the anomalous curves, indicates also to what group the curves belong. Those considered by Cardelli (1994) in his Fig. 5 all pertain to group A. No mention is made of a group of anomalous lines with a large bump, although some of its members have been studied: HD 102065, HD 103536 and HD 103875 (Boulanger et al. 1994), HD 37674 and HD 38087 (Witt et al. 1984).

Boulanger et al. (1994) have searched for a correlation between the area of the bump, i.e. $A=\pi c_{3} /(2 \gamma)$, and the warm mid-IR emission, i.e. $\nu I_{\nu}(12 \mu \mathrm{m})+\nu I_{\nu}(25 \mu \mathrm{m})$, both

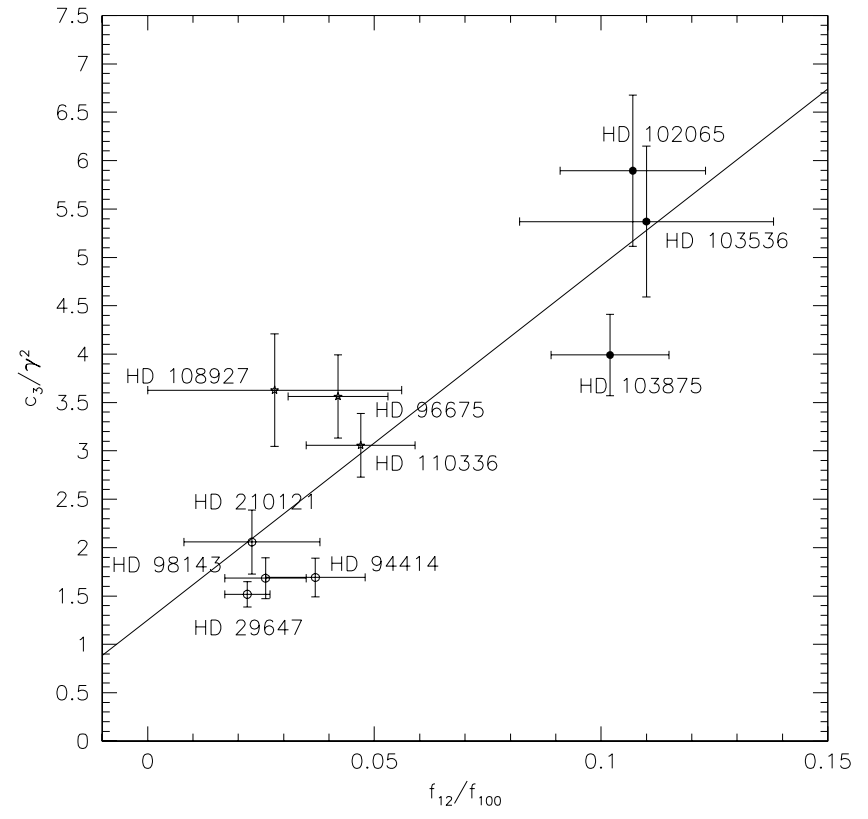

Fig. 3. Bump strength plotted against IR emission. Filled circles: group B anomalous; open circles: group A anomalous; asterisks: normal sightlines: the straight line is the least square fit of all objects

normalized to the visual extinction, $A_{V}$, towards some stars of the Chamaleon molecular complex. They claim that a quite poor correlation exists between the mentioned quantities as proved by their Fig. 5a.

We feel instead appropriate to present data for their stars in a similar, but not identical diagram.

With their data Fig. 3 has been derived in which the strength of the bump $c_{3} / \gamma^{2}$ is plotted against the ratio of the IR emission at 12 micron to that at 100 micron; error bars are computed according to Boulanger et al. (1994). Figure 3 shows a good correlation with a correlation coefficient of 0.87 . It is of particular interest the fact that the strongest emitters: HD 102065, HD 103536 and HD 103875 are type B objects, while the weakest ones belong to group A. Désert et al. (1990) have suggested that small grains of carbonaceous nature are responsible for the bump; also the mid IR emission is believed to be originated by the same type of grains.

By generalizing the behaviour here derived for the small sample of Boulanger et al. (1994) we are led to assume that the larger is the amount of warm dust responsible for the mid IR emission (i.e. $12 \mu \mathrm{m}$ ) the larger is the bump extinction. Therefore, for the same $c_{2}$ the sequence: group A anomalous, normal and group B anomalous curves can be considered as a sequence of sightlines with an increasing column density of grains of very small dimensions.

\section{Environmental characteristics}

In order to gather information on the physical nature and the behaviour of grains, the knowledge of the environment crossed by the sightlines is as much essential as the shape 


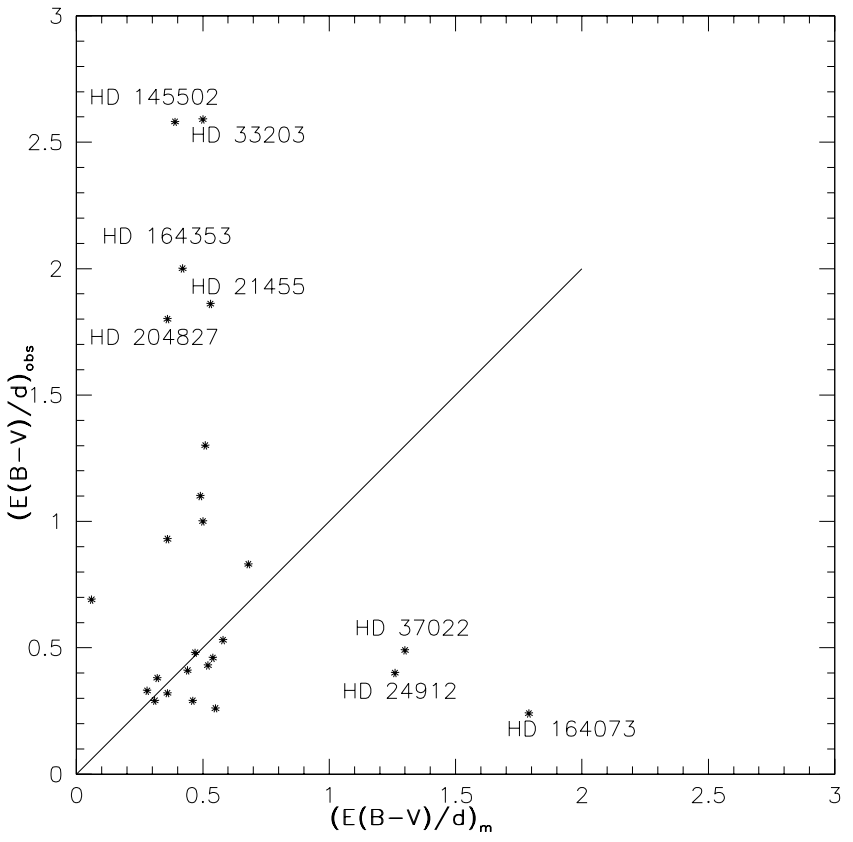

Fig. 4. Average density derived from observations compared with model estimation for anomalous curves with $d<1 \mathrm{kpc}$

of the extinction curves. Unfortunately on the first point the knowledge on environmental properties is advancing slowly, since various relevant data are still not available for many sightlines, as the column density of the different gas constituents and the depletion of the most important chemical elements.

Table 4 of Cardelli \& Clayton (1991) could help in clarifying the situation. Their data refer to two groups of sightlines classified as DC (dark cloud) and BN (bright nebulosity): the former being connected with dense clouds without nearby hot stars as is the case of VCT 10, the latter referring to sightlines affected by the presence of massive young stars $\left(\theta^{1}\right.$ Ori $\mathrm{C}$ is one example). The values of $R_{V}$ of both groups are equally scattered and overlapped. The properties of the bump and mainly its width is different for the two categories: $\gamma$ is small for $\mathrm{BN}$ objects and with a small scatter, while it is larger and with a larger scatter in the case of DC sightlines. Therefore it appears that while some features of the extinction are related to the environmental physical conditions, others seem absolutely uncorrelated. Unfortunately conclusions are often derived from too small samples. We believe that the problem of the physical and chemical properties of the interstellar matter across the different sightlines deserves further attention in order to better constrain the behaviour of the dust.

We have attempted a first approach based on the analysis of the parameter $E(B-V) / d$, where $d$ is the distance of the target star, in order to verify whether the anomalous curves are always connected with dense environments lacking massive stars (i.e. the DC sightlines of Cardelli \& Clayton 1991) or are found also in directions where the density is low. $E(B-V) / d$ is a measure of the average matter density along the sightline. The data required to
Table 6. Fraction of anomalous curves in different bins of $E(B-V)$

\begin{tabular}{|c|c|c|c|c|c|}
\hline \multicolumn{6}{|c|}{$\Delta^{2} \geq 0 . \Delta^{2} \geq 1 . \Delta^{2} \geq 2 . \Delta^{2} \geq 3 . \Delta^{2} \geq 4$} \\
\hline$E(B-V) \geq 0.10$ & 0.13 & 0.21 & 0.35 & 0.48 & 0.56 \\
\hline$E(B-V) \geq 0.20$ & 0.09 & 0.16 & 0.26 & 0.38 & 0.45 \\
\hline$E(B-V) \geq 0.30$ & 0.07 & 0.12 & 0.21 & 0.32 & 0.38 \\
\hline$E(B-V) \geq 0.40$ & 0.05 & 0.10 & 0.19 & 0.32 & 0.38 \\
\hline$E(B-V) \geq 0.50$ & 0.05 & 0.09 & 0.19 & 0.33 & 0.43 \\
\hline$E(B-V) \geq 0.60$ & 0.03 & 0.07 & 0.28 & 0.25 & 0.32 \\
\hline$E(B-V) \geq 0.70$ & 0.05 & 0.10 & 0.18 & 0.29 & 0.32 \\
\hline$E(B-V) \geq 0.80$ & 0.08 & 0.13 & 0.24 & 0.38 & 0.41 \\
\hline$E(B-V) \geq 0.90$ & 0.13 & 0.21 & 0.32 & 0.47 & 0.54 \\
\hline
\end{tabular}

compute $E(B-V) / d$ for our stars of Table 4 have been taken from the catalog of Savage et al. (1985). The error affecting such quantities is also given: when $E(B-V)$ is larger than $0.20 \mathrm{mag}$ the relative error on $E(B-V)$ is $5 \%$; the error on $d$ is about $25 \%$.

For the interpretation of $E(B-V) / d$ a model is required. We have elaborated it according to the basic concepts of Spitzer's simple model (1985) who assumes that for each sightline the column density of the gas is contributed by three components: a warm diffuse homogeneous medium, diffuse HI clouds and dense molecular clouds. We explicitly consider the first two components with parameters taken from Spitzer adopting a scale height for the diffuse gas of $200 \mathrm{pc}$ and for the HI clouds of 100 pc. We have not considered the third component, simply when the extinction per unit distance cannot be entirely accounted for by the first two components, we assume that the excess of $E(B-V) / d$ is due to the presence of molecular gas.

With this model we see that $E(B-V) / d$ is independent of the distance while it is function of the heigth over the galactic plain. Since the parameters adopted in the model are valid only for a region around the solar neighbourhood, we have restricted the consideration to sightlines referring to stars with $d<1000$ pc. In Fig. 4 the observed values of $E(B-V) / d$ are plotted against those derived by the model. There is a group of objects whose values of $E(B-V) / d$ are much larger than those of the model: this is the case of HD 21455, HD 25639, HD 33203, HD 145502, HD 164353, HD 204827 and, to a lesser extent, HD 37367, HD 169454, HD 210072, HD 217919). Their reddening cannot be interpreted without the dense molecular component. On the other hand curves like those of HD 24912, HD 37022, HD 164073 have an average density much smaller than that of the model. Possibly their lines of sight are dominated by the warm diffuse medium, lack molecular gas and cross a smaller number of $\mathrm{HI}$ clouds than expected by the model. There is another group of objects approximately characterized by a small average density both on the basis of the observed $E(B-V) / d$ and of the same quantity as derived from the model. Also for these sightlines we can infer the absence of dense gas. 
A check in the literature shows that these situations have been met. Optical molecular spectroscopy of HD 62542 and HD 29647 (Cardelli et al. 1990) suggests environments with high density (up to $10^{3} \mathrm{~cm}^{-3}$ ). According to Fitzpatrick \& Massa (1988) HD 147701 belongs to the category of sightlines crossing dense clouds. On the other hand HD 201121 is located behind the translucent cloud DBB80 (Welty \& Fowler 1992) and therefore its sightline do not cross dense regions. Clayton et al. (2000) have analysed the extinction properties in front of 30 distant (more than $1 \mathrm{kpc}$ ) galactic stars. Among them there is a group of anomalous sightlines with UV extinction curves very similar to the curves of the SMC (Gordon \& Clayton 1998), HD 62542 and HD 210121. The authors found that the average density along these lines is very low, probably due to the presence of a warm diffuse medium only.

All these arguments point towards the conclusion that the anomalous nature of the extinction curves is not necessarily tied to dense surroundings or at least that there are sightlines with anomalous extinction free of dense gas.

Another piece of information concerning the role of the environment on the character of the extinction can be obtained by analysing how the fraction of anomalous curves varies when different intervals of $E(B-V)$ and of $D^{2}$ are considered in extracting objects from the catalog of Savage et al. (1985). The same procedure described in Sect. 2 has been repeated for all the cases considered in Table 1. Table 6 has been derived correspondingly giving the fraction of anomalous sightlines for the different constraints of $E(B-V)$ and $D^{2}$. If the anomalous curves are associated only with dense environments, the fraction of these curves should decrease with decreasing reddening. This is not the case, since for low reddenings this fraction is relatively high.

Due to the relatively large number of anomalous curves we have found, a question might arise: photometric and mismatch errors can play in such a way as to transform a normal curve into an anomalous one? Let us consider the case of HD 62542 with $E(B-V)=0.33$ and $R_{V}=3.2$ (Cardelli \& Clayton 1991), whose extinction curve is one of the best studied among the anomalous ones. In order to transform it into a normal curve a vertical shift of $\delta \varepsilon_{22}=1.50$ and $\delta \varepsilon_{18}=-0.95$ is required (compare with the CCM curve with $\left.R_{V} 3.2\right)$. This implies that $E(18-V)$ and $E(22-V)$ would be affected by errors of $19 \%$ and $26 \%$ respectively. These seem too large owing to the good quality of the ANS photometry. Also mismatch errors should not be conclusive since they must act in the same direction at both wavelengths, as does any error affecting the estimate of $E(B-V)$.

\section{Conclusions}

This paper is an attempt to formulate a general analysis of the UV extinction properties based on a large number of extinction curves. Out of a sample of 252 lines of sight, selected from the catalog of Savage et al. (1985) which are characterized by shapes different from the standard curve, especially in the UV region, we have considered 132 out of them, being classified as normal or anomalous whether they fit or do not fit the CCM relations. Of them 66 strongly deviate from the relations found by CCM. On the basis of the location in the $(\Delta \epsilon)_{\mathrm{obs}}-(\Delta \epsilon)_{\mathrm{CCM}}$ diagram or in the $(\Delta \epsilon)_{\text {obs }}-\epsilon(18)$ diagram, these curves are subdivided into two groups separated by the mean of normal ones by at least $2 \sigma$ : group A and group B. The curves of the two groups differ in the strength of the bump, which, for a given $R_{V}$, is weaker than the bump of the normal curves for the former group and stronger for the latter.

By making use of the data of Boulanger et al. (1994), we find for such a small sample of sightlines that the strength of the bump correlates with the $12 \mu \mathrm{m}$ hot emission by dust. This suggests that, for a given $R_{V}$, the sequence of $\mathrm{A}$ anomalous, normals and $\mathrm{B}$ anomalous is one of increasing column density of very small grains which are responsible for both effects.

In our sample normal curves with small $R_{V}\left(R_{V} \leq\right.$ $2.80)$ and normal curves with large $R_{V}\left(R_{V} \geq 3.85\right)$ are equally numerous. While in the past little attention has been paid to the former, recent researches confirm their importance and encourage their study. It is clear that they also are important to our understanding of the dust behaviour.

The problem of the relations of the extinction properties with the environment has been also faced, however only in a preliminary way. The analysis shows that the anomaly of the extinction is not necessarily tied with dense environmental conditions, supporting existing studies of individuals curves by other authors.

\section{References}

Boulanger, F., Prévot, M. L., \& Gry, C. 1994, A\&A, 284, 956 Cardelli, J. A. 1994, ASP Conf. Ser. 58, Variability of interstellar extinction and its relationship to environment, ed. R. M. Cutri, \& W. B. Latter, 24

Cardelli, J. A., Clayton, G. C., \& Mathis, J. S. 1988, ApJ, 329, L33

Cardelli, J. A., Clayton, G. C., \& Mathis, J. S. 1989, ApJ, 345, 245 (CCM)

Cardelli, J. A., \& Savage, B. D. 1988, ApJ, 325, 864

Cardelli, J. A., Suntzeff, N. B., \& Savage, B. D. 1990, ApJ, 362,551

Cardelli, J. A., \& Clayton, G. C. 1991, AJ, 101, 1021

Carnochan, D. J. 1986, MNRAS, 219, 903

Clayton, G. C., Gordon, K. D., \& Wolff, M. J. 2000, ApJ, in press

Désert, F. X., Boulanger, F., \& Puget, J. L. 1990, A\&A, 237, 215

Draine, B. T., \& Lee, H. M. 1984, ApJ, 285, 89

Fitzpatrick, E. L., \& Massa, D. 1986, ApJ, 307, 286

Fitzpatrick, E. L., \& Massa, D. 1988, ApJ, 328, 734

Gordon, K. D., \& Clayton, G. C. 1998 ApJ, 500, 816

Jenniskens, P., \& Greenberg, J. M. 1993, A\&A, 274, 439

Larson, K. A., Whittet, D. C. B., \& Hough, J. H. 1996, ApJ, 472,755 
Li, A., \& Greenberg, J. M. 1997, A\&A, 323, 566

Massa, D., \& Savage, B. D. 1984, ApJ, 279, 320

Massa, D., Savage, B. D., \& Fitzpatrick, E. L. 1983, ApJ, 266, 662

Mathis, J. S. 1994, ApJ, 422, 176

Mathis, J. S. 1998, ApJ, 497, 824

Mathis, J. S., Rumpl, W., \& Nordsieck, K. H. 1977, ApJ, 217, 425

Mathis, J. S., \& Cardelli, J. A. 1992, ApJ, 398, 610

Meyer, D. M., \& Savage, B. D. 1981, ApJ, 248, 545

Misselt, K. A., Clayton, G. C., \& Gordon, K. D. 1999, ApJ, 515,128

Savage, B. D., \& Mathis, J. S. 1979, ARA\&A, 17,73
Savage, B. D., Massa, D., Meade, M., Wesselius, P. R., et al. 1985, ApJS, 59, 397

Seaton, M. J. 1979, MNRAS, 187, 73

Spitzer, L. 1985, ApJ, 290, L21

Snow, T. P., \& Seab, G. C. 1980, ApJ, 242, L83

Szomoru, A., \& Guhathakurta, P. 1999, preprint

Welty, D. E., \& Fowler, J. R. 1992, ApJ, 393, 193

Wesselius, P. R., van Duined, R. J., de Jonge, A. R. W., et al. 1982, A\&AS, 49, 427

Witt, A. N., Bohlin, R. C., \& Stecher, T. P. 1984, ApJ, 279, 698

Whittet, D. C. D., \& van Breda, I. G. 1980, MNRAS, 192, 467 